\title{
Traqueostomía percutánea por dilatación sin fibrobroncoscopio en la Unidad de Cuidados Intensivos del Hospital Carlos Alberto Seguín Escobedo, Arequipa 2011-2014
}

\section{Percutaneous dilational tracheostomy without using a bronchoscope in the Intensive Care Unit of Carlos Alberto Seguin-Escobedo National Hospital, Arequipa 2011-2014}

Correspondencia Rafael Tapia Pérez rafaeltapiap@gmail.com

Recibido: 22/11/2016 Arbitrado por pares Aprobado: 28/02/2017

Citar como:Tapia-Pérez $R$, Barredade la Cruz M, Alcázar-Zuzunaga $P$, Fajardo-Karlo L, Oporto-Gonzales F, Pérez Vargas Y. Traqueostomía percutánea por dilatación sin fibrobroncoscopio en la Unidad de Cuidados Intensivos del Hospital Carlos Alberto Seguín Escobedo, Arequipa 2011-2014. Acta Med Peru. 2017:34(1):27-32
Rafael Tapia-Pérez ${ }^{1,2}$, Miguel Barreda-De La Cruz ${ }^{1}$, Pedro Alcázar-Zuzunaga1, Luis Fajardo-Karloํㅜ, Fabricio Oporto-Gonzales' ${ }^{1}$, Yvan Pérez-Vargas ${ }^{1}$

1 Unidad de Cuidados Intensivos, Hospital Nacional Carlos Seguín Escobedo. Arequipa, Perú.

2 Facultad de Medicina Humana, Universidad Católica Santa María. Arequipa, Perú.

\section{RESUMEN}

Objetivo: Describir la experiencia de traqueostomía percutánea por dilatación (TPD) sin fibrobroncospio en la Unidad de Cuidados Intensivos (UCI) del Hospital Nacional Carlos AlbertoSeguín Escobedo (EsSalud), Arequipa. Materiales y métodos: Estudio restrospectivo y descriptivo. Se recolectaron los registros de pacientes desde el 2011 hasta el 2014, a los que se realizó TPD sin fibrobroncoscopio. Se evaluaron variables demográficas, tiempo de realización de las TPD, diagnóstico de ingreso, comorbilidades asociadas, APACHE II, indicaciones de traqueostomía y complicaciones. Resultados: De las 94 TPD, el 48.9\% fueron varones; la edad promedio fue $60,3 \pm 19,7$ años; el $35,1 \%$ procedieron del servicio de emergencia; el score APACHE II fue de $18,1 \pm 5,8$ puntos. Se estimó un tiempo promedio de $14,6 \pm 12,6$ días para la realización de las traqueostomías. Al $97,9 \%$ se les realizó traqueostomía tardía. A 17 pacientes $(18,1 \%)$ se les practicó una ecografía cervical; el $14,9 \%$ tuvieron como diagnóstico de ingreso a la UCI TEC grave; la mayor indicación fue la falla neurológica con el $47,8 \%$. Las complicaciones perioperatorias immediatas no mortales fueron: hemorragia leve, infección del estoma, hipotensión, enfisema subcutáneo y falsa vía. Se presentó una complicación mortal por hemorragia severa relacionada al procedimiento y la complicación tardía más frecuente fue estenosis subglótica. Finalmente, se halló una sobrevida global del $71,3 \%$. Conclusiones: La TPD mediante la técnica Ciaglia Blue Rhino sin la asistencia de fibrobroncoscopio y a la cabecera del paciente sería un procedimiento seguro y con baja incidencia de complicaciones severas.

Palabras clave: Traqueostomía, dilatación. (Fuente: DeCS BIREME). 


\begin{abstract}
Objective: To describe the experience with percutaneous dilational tracheostomy (PDT) without using a bronchoscope in the Intensive Care Unit (ICU) of Carlos Alberto Seguin-Escobedo National Hospital, EsSalud, Arequipa. Materials and methods: This is a retrospective and descriptive study. Records of patients who underwent PDT without a bronchoscope from 2011 to 2014 were collected. Demographic variables, time of TPD completion, diagnosis at admission, comorbidities, APACHE II score, indications for tracheostomy, and complications were assessed. Results: Of 94 PDT procedures performed, $48.9 \%$ were in male subjects; the average age of patients was $60.3 \pm 19.7$ years; more than one third (35.1\%) were brought from the Emergency Service; the APACHE II score was $18.1 \pm 5.8$ points. An average time of $14.6 \pm 12.6$ days elapsed before the procedures were performed. Most patients (97.9\%) underwent a late tracheostomy. Seventeen (18.1\%) patients had a previous neck ultrasonography performed; $14.9 \%$ had severe head trauma as an admission diagnosis to the ICU; the main indication for admission was neurological failure in nearly half of the patients (47.8\%). Perioperative immediate non-lethal complications were: minor bleeding, stoma infection, hypotension, subcutaneous emphysema and false passage. There was one fatal complication, severe bleeding related to the procedure, and the most frequent late complication was subglottic stenosis. Finally, the overall survival rate was $71.3 \%$. Conclusions: PDT using the Ciaglia Blue Rhino technique without bronchoscopic assistance performed at the bedside would be a safe procedure with a low incidence of severe complications.
\end{abstract}

Keywords: Tracheostomy, dilatation (Source: MeSH NLM).

\section{INTRODUCCIÓN}

En el año 1985, Ciaglia introdujo la traqueostomía percutánea por dilatación (TPD) y en 1999 el mismo autor mejoró su técnica con el método denominado como Ciaglia Blue Rhino de un solo paso. Esta técnica es un procedimiento fácil y seguro para acceder a la vía aérea, y es empleada sobre todo para aquellos pacientes con ventilación mecánica prolongada en la Unidad de Cuidados Intensivos (UCI) ${ }^{[1]}$.

Así, la TPD se ha convertido en una verdadera alternativa a la técnica quirúrgica convencional, siendo incluso de primera elección en pacientes críticos; así vemos que se llega a realizar hasta en el $78,4 \%$ de $\mathrm{UCl}$ en algunos países ${ }^{[2]}$.

Entre sus múltiples ventajas se tiene que es un procedimiento mínimamente invasivo, presenta un alto grado de seguridad, es de fácil realización, necesita de una incisión pequeña, tiene bajo riesgo de sangrado, requiere un tiempo quirúrgico corto, tiene bajo costo $y$, sobre todo, puede realizarse a la cabecera del paciente sin requerir un quirófano; desde el punto de vista funcional, ofrece menor resistencia al flujo aéreo, reduce el trabajo respiratorio y mejora la mecánica respiratoria del paciente, facilitando así, el destete de la ventilación mecánica ${ }^{[3,4]}$.

Por otro lado, acorta el tiempo de hospitalización en $\mathrm{UCl}$, garantiza una nutrición adecuada, autonomía precoz, confort y mejor comunicación del paciente. Asimismo, se ha reportado una mejor tolerancia que el tubo endotraqueal; permite reducir la administración de sedoanalgesia; reduce el daño laríngeo y lo más relevante, reduce la aparición de neumonía asociada a ventilación mecánica (NAV) cuando se realiza de forma precoz ${ }^{[3-5]}$.

Las indicaciones de la TPD en UCI son diversas, siendo las principales: ventilación mecánica prolongada, lesiones neurológicas persistentes o permanentes, problemas neuromusculares, distrés respiratorio agudo prolongado 0 complicado y procesos obstructivos de las vías aéreas ${ }^{[5-7]}$. La TPD también ha resultado ser costo-efectiva respecto la traqueostomía quirúrgica. Higgins y Punthakee concluyeron en el 2007 que la TPD resultaba "favorable" en 457 dólares frente a la traqueostomía quirúrgica ${ }^{[4,6,7]}$.

La TPD como cualquier otro procedimiento presenta contraindicaciones, limitaciones, riesgos y complicaciones. Entre las complicaciones tenemos sangrado perioperatorio, infecciones, estenosis traqueal, neumotórax, perforación esofágica, enfisema subcutáneo, fractura del anillo traqueal y obstrucción de la vía aérea ${ }^{[3-5]}$.

En la Unidad de Cuidados Intensivos del Hospital Nacional Carlos Alberto Seguín Escobedo - EsSalud, Arequipa, desde el 2011 se viene realizando la traqueostomía percutánea por dilatación sin fibrobroncospía, técnica que nos permite otorgar apoyo respiratorio a los pacientes críticos con la finalidad de disminuir los riesgos de daño asociado al tubo orotraqueal, facilitar el manejo de la vía aérea y el destete de la ventilación mecánica. El objetico de nuestro estudio es describir la experiencia de la TPD sin fibrobroncoscopía (TPDSF) a la cabecera del paciente y evaluar los beneficios y complicaciones del mismo.

\section{MATERIAL Y MÉTODOS}

Estudio descriptivo y retrospectivo. La población de estudio comprendió a todos los pacientes a los que se les realizó TPDSF en la UCl del Hospital Nacional Carlos Alberto Seguín Escobedo de la ciudad de Arequipa-Perú, independientemente de su causa o patología de fondo, durante el periodo de enero de 2011 a diciembre 2014. Todas las TPD fueron electivas y se realizaron por el equipo de $\mathrm{UCl}$ a la cabecera del paciente de manera estandarizada de acuerdo a lo establecido por la técnica de 
Ciaglia Blue Rhino, usando el set de TPDSF y bajo monitoreo electrocardiográfico, hemodinámico y ventilatorio.

Se incluyeron a todos los pacientes tributarios de TPDSF. Se excluyeron a pacientes con traqueostomía a cielo abierto, ser menor de 18 años, tener el diagnóstico de obesidad mórbida o presentar alguna tumoración de cuello.

\section{Técnica Quirúrgica}

Paciente bajo sedoanalgesia con propofol y fentanilo, con monitoreo hemodinámico y ventilatorio $y$, con tres operadores: uno controlando la vía aérea y los otros realizando la TPDSF. En algunos casos se recurrió a la ecografía cervical para verificar la posición de la tráquea y facilitar el procedimiento.

Bajo asepsia y antisepsia estrictas, se identificó el espacio a dos traveses de dedo por encima de la escotadura esternal, aproximadamente entre el tercero y cuarto anillo traqueal. Se procedió a infiltrar lidocaína al $2 \%$ y a realizar la técnica de TPDSF en un solo paso. Previamente el operador del control respiratorio retiró el tubo orotraqueal hasta 17 a $18 \mathrm{~cm}$ permitiendo el acceso de la vía traqueal. La ventilación mecánica se mantuvo con una $\mathrm{FiO}_{2}$ de $100 \%$ y niveles bajos de presión positiva al final de la expiración (PEEP). Luego del procedimiento, se tomó radiografía de tórax para verificar posicionamiento del tubo de traqueostomía.

Se utilizó una ficha de datos donde se registraron todas las variables de aquellos pacientes a los que se le realizóTPDSF, que incluyó edad, sexo, diagnóstico de ingreso a la UCI, puntuación de Acute Physiology and Chronic Health Evaluation II (APACHE II), indicación para la TPDSF, tiempo de ventilación mecánica, complicaciones intra y postoperatorias y, tiempo de realización de laTPDSF. LaTPDSF se consideró precoz si fue realizado hasta los siete días y tardío después de los siete días del ingreso a UCI.

\section{RESULTADOS}

Durante el período de estudio se realizaron 94TPDSF. En cuanto al sexo, el $51,1 \%$ fueron mujeres ( $p>0,05$ entre sexos). La edad promedio fue $60,3 \pm 19,7$ años (rango: 18-92 años). El 35,1\% de los pacientes a los que se realizaron el procedimiento de TPDSF fueron admitidos por el servicio de emergencia (Tabla 1). La puntuación promedio APACHE II observada a las 24 horas del ingreso fue de 18,1 puntos (rango: 12 a 34 puntos). Por otro lado, se encontró que el $14,9 \%$ de los pacientes a los que se realizóTPD tuvieron como diagnóstico de ingreso a UCl de trauma encéfalo craneano (TEC) grave (Tabla 2).

Desde la intubación orotraqueal hasta la realización de la TPDSF se observó un tiempo promedio de $14,6 \pm 12,6$ días (rango: 3 a 39 días). Al 97,9\% de los pacientes se les realizó la TPDSF tardía (Tabla 3). Cabe precisar que solo a $17(18,1 \%)$ pacientes se les realizó ecografía cervical anterior como ayuda para dirigir el procedimiento (Tabla 3).

Se observó que la falla neurológica $(47,8 \%)$ fue el diagnóstico más frecuente que llevó a la realización de TPDSF (Tabla 3).

Tabla 1. Características de pacientes con traqueostomía percutánea por dilatación.

\begin{tabular}{lc}
\multicolumn{1}{c}{ Características } & $\mathbf{n}(\%)$ \\
Sexo & \\
Hombres & $46(48,9)$ \\
Mujeres & $48(51,1)$ \\
Grupo etario (años) & \\
$18-30$ & $11(11,7)$ \\
$31-45$ & $9(9,6)$ \\
$46-60$ & $23(24,5)$ \\
$61-75$ & $25(26,5)$ \\
$76-90$ & $23(24,5)$ \\
$>90$ & $3(3,2)$ \\
Edad (años) & $19,7($ rango: 18 a 92$)$ \\
Servicio de procedencia & \\
Emergencia & $33(35,1)$ \\
Neurocirugía & $20(21,3)$ \\
Medicina interna & $15(15,9)$ \\
Neurología & $14(14,9)$ \\
Otros & $12(12,8)$ \\
\hline
\end{tabular}

Aquí se incluye TEC grave, enfermedad vascular cerebral (EVC) hemorrágico, EVC isquémico y síndrome posparo cardiorrespiratorio.

Las complicaciones perioperatorias inmediatas no mortales observadas en los pacientes en estudio fueron: hemorragia leve, infección de estoma, hipotensión, enfisema subcutáneo y falsa vía. Se resalta que el sangrado leve constituyó la complicación

Tabla 2. Diagnóstico de ingreso a Unidad de Cuidados Intensivos por traqueostomía percutánea por dilatación $(n=94)$

Diagnóstico de ingreso

TEC grave

Patología infecciosa pulmonar

EVC hemorrágico

Otras patologías del SNC

Sepsis

Patología quirúrgica

EVC isquémico

Pancreatitis aguda

EPOC/Asma bronquial

Síndrome post PCR

Traumatismos

Misceláneo

EVC: Evento vascular cerebral; PCR: Paro cardiorrespiratorio

TEC: Traumatismo cráneo-encefálico; SNC: Sistema nervioso central

\section{$\%$} 14,9 15,9 11,7 11,7 12,8 6,4 6,4 4,3 4,3 3,2 4,3 9,6 
Tabla 3. Indicaciones, tiempo para la ejecución y traqueostomía percutánea por dilatación bajo ecografía $(n=94)$.

\section{N}

$\%$

\section{Indicación para realización TPD}

$\begin{array}{lll}\text { Falla neurológica } & 45 & 47,8 \\ \text { Ventilación mecánica prolongada } & 37 & 39,4 \\ \text { Otros } & 12 & 12,8\end{array}$

Tiempo para la ejecución TPD $\quad 14,6 \pm 12,6 \quad 3-39$ días

\begin{tabular}{lcc} 
Momento de la TPD & & \\
Siete días a menos & 2 & 2,1 \\
Después de los siete días & 92 & 97,9 \\
TPD bajo visión con Ecografía & 17 & 18,1 \\
Tiempo operatorio & $13,0 \pm 9,4$ (5-30 minutos) \\
\hline
\end{tabular}

intraoperatoria más frecuente con $12(12,7 \%)$ de casos. Sin embargo, se describe un paciente fallecido en el intraoperatorio por hemorragia severa relacionada al procedimiento. La complicación tardía más frecuente después de las cuatro semanas fue la estenosis subglótica en dos casos (Tabla 4).

Complementariamente, la comorbilidad más frecuente fue la hipertensión arterial, encontrada en $38(40,4 \%)$ pacientes, seguido de la diabetes mellitus tipo II $(20,2 \%)$ y los problemas crónicos de las vías respiratorias (13,8\%). El 71,3\% (67/94) de los pacientes a los que se realizó TPDSF sobrevivieron y fueron dados de alta de UCI. La diferencia $(28,7 \%)$ falleció por razones no relacionadas al procedimiento.

\section{DISCUSIÓN}

La mayor cantidad de TPDSF fueron realizadas en mujeres con $51,1 \%$ y sin diferencias significativas respecto a los varones; $\sin$ embargo, comparando con estudios realizados anteriormente, existen trabajos donde predominan los varones y otro que concuerda con nuestros resultados ${ }^{[8]}$. Este hallazgo no tiene mayor importancia y dependerá de la patología de fondo que condicione los criterios para realizar la TPDSF.

De la misma forma, podemos observar que la edad promedio de los pacientes a los que se les realizó TPDSF, fue de 60,3 \pm 19,7 años de edad, encontrándose un amplio rango de edad. De este modo encontramos 1 paciente de 18 años y 3 pacientes mayores de 90 años. Comparando con la bibliografía, en algunos estudios se observó que la mayoría de los pacientes fueron adultos mayores ${ }^{[9,10]}$, en cambio, en otras revisiones había una tendencia de edades que iban de 25 a 55 años ${ }^{[11-13] ;}$; es decir, una población económicamente activa. En nuestro estudio, las patologías condicionantes se presentan con más frecuencia en pacientes mayores.

Sabemos que las complicaciones de la traqueostomía quirúrgica son dos a tres veces más frecuentes que la técnica percutánea y varían entre 6,7 y $48,0 \%$, con una mortalidad del 1,6\% [7,9,14${ }^{16]}$. En general, se puede afirmar que la morbilidad de la TPD es baja, pero la cifra exacta varía enormemente según los autores consultados, entre el $5 \%$ y $25 \%$, llegando incluso, al $3 \%$ con la técnica de Ciaglia Blue Rhino ${ }^{[2]}$. En nuestro estudio, se muestra una morbilidad global del $34 \%$, que comprende las complicaciones perioperatorias inmediatas y tardías. Sólo hubo un caso de complicación fatal debido a sangrado. Estos hallazgos son similares a otros estudios ${ }^{[1,12,17]}$.

También podemos observar que la mayor proporción de pacientes a los que se realizó TPD, proceden del Servicio de Emergencia, seguido del servicio de Neurocirugía y Neurología, grupo de pacientes especiales, que por tener comprometido el sistema nervioso central (SNC) y/o periférico (SNP), son proclives a la ventilación mecánica prolongada $\mathrm{y}$, por tanto, susceptibles de TPD.

En la UCl de nuestro hospital, se evalúa la puntuación de APACHE II, a todos los pacientes dentro de las primeras 24 horas de ingreso, escala que valora la severidad de su condición patológica y de la misma forma puede predecir el pronóstico de mortalidad. Nosotros hemos trabajado con un promedio de 18,1 $\pm 5,8$ puntos a los pacientes a los que se les realizó TPD, con un rango variable que va entre 12 y 34 puntos. Este promedio encontrado establece una probabilidad de mortalidad de 29,6 a $36,7 \%$. Ahora bien, nosotros diferenciamos la mortalidad asociada directamente a la TPD de la mortalidad global no relacionada con la técnica de TPD; es decir, de sus patologías subyacentes, en donde se observó una mortalidad del $28,7 \%$. Este hallazgo se semeja con otros estudios donde la mortalidad de pacientes traqueostomizados es de $36 \%$, aunque curiosamente tuvieron un score APACHE II más bajo, con promedios de 15 puntos al ingreso de la $\mathrm{UCl}[3,4,18-20]$.

El tiempo promedio estimado para la realización de TPD por dilatación, desde la intubación orotraqueal, fue de $14,6 \pm$ 12,6 días, existiendo un amplio rango que osciló entre 3 y 39 días. La bibliografía muestra reportes controversiales sobre la definición de traqueostomía (TOO) precoz y tardía desde la intubación endotraqueal. Por tal motivo, elegimos la propuesta de traqueostomía precoz cuando la TPDSF se realiza hasta los 7 días, y tardía cuando se realiza después de los 7 días ${ }^{[21]}$. En nuestro trabajo observamos que en el $97,9 \%$ de los pacientes, se les realizó una traqueostomía tardía; las razones de esta conducta se debieron a diferentes factores siendo las más importantes la patología de fondo, la evolución del cuadro clínico y motivos de logística (falta de sets de TQO). Por otro lado, en solo dos pacientes $(2,1 \%)$ se realizó TPDSF precoz. Sobre este aspecto, planteamos que en virtud a este procedimiento que se ha vuelto rutinario en toda $\mathrm{UCl}$, debe establecerse un consenso definitivo sobre traqueostomía precoz y tardía sobre la base de pronóstico, beneficios y riesgos. En los estudios revisados se encontró que los promedios de tiempos para la realización de traqueostomías varían entre 8 a 15 días, teniendo mucha correlación con lo encontrado en nuestro trabajo ${ }^{[10,22-25]}$.

Resaltamos el hecho que en la mayoría de TPDSF realizadas en nuestro servicio, no hubo necesidad de técnicas o medios 
de ayuda para el procedimiento; sin embargo, en $18,1 \%$ pacientes, se realizó ecografía de cuello para ubicar de manera más precisa la tráquea y la luz endotraqueal, es decir realizar una TPD guiada por ecografía ${ }^{[26-28]}$. Esta técnica no se realizó en todos los pacientes, porque no todos los operadores tenían experiencia en ecografía, por lo que la TPD fue a "ciegas", usando solo parámetros anatómicos y fisiológicos. De otra forma, mencionamos, que en ningún caso usamos como técnica de apoyo a la fibrobroncoscopía y no encontramos mayores complicaciones o limitaciones ${ }^{[3,11,29-31]}$. En suma, la fibrobroncospía y la ecografía no resultaron importantes para la ejecución de TPD ${ }^{[32]}$.

En $14,9 \%$ pacientes con TPDSF tuvieron como diagnóstico de ingreso TEC grave; por otro lado, la causa compartida más frecuente fue la patología infecciosa pulmonar que comprende la neumonía nosocomial y el distrés respiratorio. También resaltamos que los EVC tanto hemorrágicos como isquémicos se observaron en un $11,7 \%$ y $6,4 \%$ respectivamente. Esto tiene importancia porque la suma de todos los problemas relacionados a falla neurológica constituyó el $47,8 \%$ de los casos estudiados ${ }^{[4,33,34]}$. De esto se infiere que independientemente de una patología particular, los problemas neurológicos son la causa más importante de ventilación mecánica prolongada y por tanto susceptibles de TPD.

Existe otro grupo de patologías que también se asocian a ventilación mecánica prolongada y se caracterizan por comprometer la función respiratoria como la pancreatitis aguda, las enfermedades pulmonares crónicas descompensadas, la sepsis, patologías quirúrgicas, entre otros. Por otro lado, la comorbilidad más frecuente fue la hipertensión arterial, hallada

Tabla 4. Complicaciones perioperatorias inmediatas y tardías. n

Complicaciones inmediatas $(n=27)$

Hemorragia leve 12 Infección de estoma 5

Hipotensión

Enfisema subcutáneo

Falsa vía

Hemorragia moderada

Hemorragia severa

Traqueostomía fallida

Mortalidad operatoria

Complicaciones tardías $(n=5)$

Estenosis subglóticas

Estenosis traqueal

Fístulas traqueoesofágicas

Granuloma laríngeo

\section{Complicaciones fatales}

$\%$

12,7

5,3

2,1

2,1

2,1

1,1

1,1

1,1

1,1
*Total de TPD $=94$

Hemorragia leve: $<20$ cc o que cede espontaneamente

Hemorragia moderada: >20 cc o que requiere compresión o sutura Hemorragia severa: Disminución de hematocrito $>3$ puntos en 38 pacientes, seguido de la diabetes mellitus y de problemas respiratorios crónicos ${ }^{[8,34,35]}$. Estas entidades nosológicas toman relevancia en la medida que pueden provocar complicaciones; sin embargo, es poco significativo la asociación con TPD, lo que no es observado en otros estudios. A medida que alcanzamos destreza en el procedimiento, el tiempo operatorio fue mejorando; tal es así, que el tiempo promedio operatorio fue de 13 minutos con un rango de 5 a 30 minutos.

En cuanto a las complicaciones perioperatorias inmediatas observamos la ocurrencia en $28,7 \%$ de casos. La Hemorragia leve, considerada como aquella que presenta una pérdida sanguínea de menos de $20 \mathrm{~cm}^{3}$ o que cede espontáneamente, es la primera complicación encontrada en nuestro estudio en 12 casos $(12,7 \%)$. Complicación que se correlaciona proporcionalmente con las investigaciones reportadas ${ }^{[10,29,35]}$. Así mismo, hallamos otras complicaciones como infección del estoma, hipotensión, enfisema subcutáneo, falsa vía y hemorragia moderada. Sin embargo, debemos subrayar que tuvimos un caso $(1,1 \%)$ con hemorragia severa que a su vez constituyó la única muerte reportada durante la realización de la TPD. También subrayamos que este caso no fue bajo ecografía.

Nosotros reportamos 5,3\% casos de complicaciones tardías, siendo la estenosis subglótica la complicación tardía más frecuentemente observada, resultado que se correlaciona también con los hallazgos de trabajos previos $[5,8,21,21]$.

Por otro lado, la mortalidad global observada, como fue descrita líneas arriba, no está relacionada con la TPD, y la sobrevida global por la enfermedad de origen fue observada en $67(71,3 \%)$ pacientes.

En conclusión, la TPD mediante la técnica Ciaglia Blue Rhino sin la asistencia de fibrobroncoscopio y a la cabecera del paciente sería un procedimiento seguro y con baja incidencia de complicaciones severas.

Fuentes de financiamiento: autofinanciado.

Conflictos de interés: los autores declaran no tener ningún conflicto de interés.

\section{REFERENCIAS BIBLIOGRÁFICAS}

1. Paez M, Buisán F, Almaraz A, Muñoz F. Traqueotomía percutánea con la técnica de Ciaglia Blue Rhino: un análisis crítico tras un año de experiencia. Rev Esp Anestesiol Reanim. 2005;52(8):466-73.

2. Rodríguez LC, Llinás Alvarez L. Guía de traqueostomía percutánea. GM-UIA-005. Vía Puerto, Colombia: Hospital Universidad del Norte; 2012.

3. Calvache JA, Molina García RA, Trochez AL, Benitez F, Arroyo L. Traqueostomía percutánea por dilatación sin fibrobroncoscopio. Evaluación de 80 casos en cuidados intensivos. Rev Colomb Anestesiol. 2013;41(3):184-9.

4. Martínez Hurtado E, Olivas García E, Martínez Fariña P, Bernardino Santos M, García del Valley Manzano S. Traqueotomía Percutánea en Reanimación (I): Historia, indicaciones, complicaciones. Rev electron AnestesiaR [Internet]. 2012 [citado el 12 de enero de 2017];4(4):176. Disponible en: http://anestesiar.org/rear/ 
volumen-4/numero-04/347--traqueotomia-percutanea-enreanimacion-i-historia-indicaciones-complicaciones.html

5. Ramírez-Arce J, Padilla-Cuadra JI, Sánchez-Arias M. Traqueostomía percutánea por dilatación: Reporte de 70 casos. Acta Méd Costarric. 2006;48(2):77-83.

6. Añón JM, Araujo JB, Escuela MP, González-Higueras E. Traqueotomía percutánea en el paciente ventilado. Med Intensiva. 2013;38(3):181-93.

7. Higgins K, Punthakee X. Comparación metaanálisis de traqueostomia abierta versus percutánea. Rev Otorrinolaringol Cir Cabeza Cuello. 2007;67(1):81-4.

8. Pérez $E$, Yañez R, Avilés $E$, Alarcón $C$, Weisse $O$, Curi $M$, et al. Traqueostomía percutánea en una unidad de cuidados intensivos. Rev Chil Cir. 2011;63(4):356-60.

9. Asus C, Bustos V, Vega M, Yalour JC, Alabarse G. Traqueostomía percutánea versus convencional. Rev Argent Resid Cir. 2009;13(2):76-8.

10. Vásquez J, Fang-Li J, Arauco V. Traqueostomía percutánea de un solo paso en las unidades de cuidados intensivos e intermedios del Hospital Nacional Almanzor Aguinaga Asenjo. Rev cuerpo méd HNAAA. 2010; 3(1):1-8.

11. Celedón C, Walker K, Naser A, Neumann P, Nazar R. Traqueostomía abierta vs traqueostomía percutánea. Rev Otorrinolaringol Cir Cabeza Cuello. 2007; 67(3): 222-8.

12. Marcel S, Metschke M, Braune SA, Püschel K, Kluge S. Death after percutaneous dilatational tracheostomy: a systematic review and analysis of risk factors. Crit Care. 2013;17(5): R258.

13. Da Silva R, Avertano A, Youndes R. [Percutaneous Tracheosthomy: ideal technique?]. Rev Col Bras Cir. 2011; 38(6): 1-8. Portuguese

14. De la Garza H, Maycotte S, Durán L, Álvarez R, Estrada G. Traqueotomía por dilatación percutánea vs traqueotomía abierta. Experiencia en el Hospital Ángeles Lomas. AN ORL MEX 2011; 56 (3).

15. Orjuela O, Santofimio D, Lastra G, Rivera L. Traqueostomía percutánea versus traqueostomía abierta en el paciente crítico. Acta Colomb Cuid Intensivo 2011; 11(1): 3-11.

16. Delaney A, Bagshaw SM, Nalos M. Percutaneous dilatational tracheostomy versus surgical tracheostomy in critically ill patients: a systematic review and meta-analysis. Crit Care. 2006;10(2):R55.

17. Glossop AJ, Meekings T, Hutchinson S, Webber S. Complications following tracheostomy insertion in critically ill patients experience from a large teaching hospital. JICS. 2011;12(1):301-6.

18. Shaheen I. Percutaneous Tracheostomy: Who is not a Candidate? Clin Res Pulmonol. 2013;1:1009-11.

19. Gutiérrez C, Aguilera G, Fajardo M. Traqueostomía dilatacional percutánea. Descripción de la técnica. Rev Cub Med Int Emerg. 2004; 3(4) 124-134

20. Australian New Zealand intensive Care Society. Percutaneous Dilatational Tracheostomy Consensus Statement. New Zealand: ANZICS; 2010.

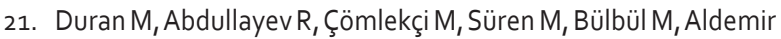
T. Comparison of early and late percutaneous tracheotomies in adult intensive care unit. Rev Bras Anestesiol. 2014;64(6):43842.

22. Engels PT, Bagshaw SM, Meier M, Brindley PG. Tracheostomy: from insertion to decannulation. Can J Surg. 2009;52(5):427-33.

23. Esteves $\mathrm{C}$ y col. Traqueotomía y Traqueostomía. Rev Anestesiol. 2008: $24(2): 24-32$.

24. Gaviria E, Cuervo F, Arango G, Arroyave H, Quiceno I, Puerta J. Traqueostomía percutánea por dilatación: experiencia con 30 pacientes electivos en Unidad de Cuidados Intensivos (UCI). Rev Colomb Anestesiol. 2002;30(4):1-7.

25. Romero C, Cornejo R, Tobar E, Pablo O, Gálvez R, Espinoza M, et al. Traqueostomía percutánea en la Unidad de Cuidados Intensivos. Rev Chil Med Intensiva. 2009;24(4): 215-22.

26. Zamudio-Burbano $M$, Casas-Arroyave F. El uso del ultrasonido en el manejo de la vía aérea. Rev Colomb Anestesiol. 2015;43(4):307-13.

27. Martínez-Hurtado E. Traqueotomía percutánea con seguridad para el paciente crítico: checklist, ecografía y fibrobroncoscopio. 21 nov 2011 [citado el 12 ene 2017]. En: Anestesiar.org [Internet]. España: Asociación Anestesia Reanimación España. Disponible en: http:// anestesiar.org/2011/traqueotomia-percutanea-con-seguridad-parael-paciente-critico-ii-checklist-ecografia-y-fibrobroncoscopio/

28. Yavuz A, Yılmaz M, Göya C, Alimoglu E, Kabaalioglu A. Advantages of US in percutaneous dilatational tracheostomy: randomized controlled trial and review of the literature. Radiology. 2014;273(3):927-36.

29. Álvarez-Maldonado P, Pérez-Rosales A, Nuñez-Pérez C, CuetoRobledo G, Navarro-Reynoso F, Cicero-Sabido R. Traqueostomía percutánea guiada por broncoscopia en cuidados intensivos. Cirugia y cirujanos. 2013;81(2):92-7.

30. Sanabria L, Romero E, Chávarro O, Santofimio D. Impacto de la implementación de la traqueostomía percutánea en la UCI-A del Hospital Universitario de Neiva. Revista Facultad de Salud. 2009; 1(2): 39-47.

31. Marullo L, Izzo G, Orsini A, Petruzzi J, d'Elia A, Vessichio L. Clinical features as discriminating factors in the choice of tracheostomy techniques. BMC Surg. 2013;13(Suppl 1):A28.

32. Gobatto AL, Besen BA, Tierno PF, Mendes PV, Cadamuro F, Joelsons $D$, et al. Ultrasound-guided percutaneous dilational tracheostomy versus bronchoscopy-guided percutaneous dilational tracheostomy in critically ill patients (TRACHUS): a randomized noninferiority controlled trial. Intensive Care Med. 2016;42(3):342-51.

33. Fikkers BG, et al. Tracheostomy on the intensive care unit for adult patients. Guidelines Tracheostomy of the NVIC. 2013; 1: 1-20.

34. Hernández C, Bergeret J, Hernández M. Traqueostomía: principios y técnica quirúrgica. Cuad Cir. 2007;21:92-8.

35. Salcedo O, Frutos-Vivar F. Traqueostomía en pacientes ventilados: ¿para qué la hacemos? Med Intensiva. 2008;32(2):91-3. 